\title{
Les configurations de l'espace, du temps et de la subjectivité dans un contexte de terreur : l'exemple colombien
}

\section{Daniel Pécaut}

\section{(2) OpenEdition Journals}

\section{Édition électronique}

URL : http://journals.openedition.org/conflits/393

DOI : 10.4000/conflits.393

ISSN : $1777-5345$

Éditeur :

CCLS - Centre d'études sur les conflits lilberté et sécurité, L'Harmattan

Édition imprimée

Date de publication : 1 mars 2000

ISBN : 2-7384-9385-8

ISSN : 1157-996X

\section{Référence électronique}

Daniel Pécaut, « Les configurations de l'espace, du temps et de la subjectivité dans un contexte de terreur : l'exemple colombien », Cultures \& Conflits [En ligne], 37 I printemps 2000, mis en ligne le 20 mars 2006, consulté le 30 mars 2021. URL : http://journals.openedition.org/conflits/393 ; DOI : https://doi.org/10.4000/conflits.393

Ce document a été généré automatiquement le 30 mars 2021.

Creative Commons License 


\title{
Les configurations de l'espace, $\mathrm{du}$ temps et de la subjectivité dans un contexte de terreur : l'exemple colombien
}

\author{
Daniel Pécaut
}

1 Dans un article publié dans cette même revue, j'avais relevé que la violence récente en Colombie a longtemps été perçue comme un phénomène banal ${ }^{1}$. Elle était présentée comme la continuation de la violence des années 1950, voire d'une violence qui aurait en permanence caractérisé l'histoire colombienne. On considérait que, si elle faisait des victimes, elle n'avait pour le pays qu'un coût limité et comportait aussi des bénéficiaires, ceux qui profitaient de ses retombées économiques qui, trafic de la drogue aidant, n'étaient pas négligeables et suscitaient un marché du travail alternatif ${ }^{2}$. J'indiquais dans cette même étude que la banalité était en train de s'estomper et que, notamment dans les régions où prévalaient désormais les confrontations entre divers protagonistes armés, elle faisait place à une terreur qui ne laissait plus d'autre choix à la population civile que de partir ou de s'adapter à un contexte de défiance généralisée en se repliant sur des stratégies individualistes de survie.

2 Entre une situation banale de violence et une situation de terreur, la différence vient de ce que les principes normatifs partagés et les références institutionnelles qui, dans un cas, subsistent au moins en arrière-plan tendent à s'effacer dans l'autre. Le passage à la terreur correspond au moment où ses protagonistes recourent à des moyens qui visent à briser les liens sociaux qui définissaient la particularité de secteurs donnés de la population, secteurs qui se trouvent désormais soumis à une emprise contre laquelle ils ne peuvent appeler à aucun tiers, à aucune institutionnalité reconnue.

Dans le cas colombien, la terreur ne prend pas la forme d'une action de l'Etat contre certaines populations. Le régime se réclame toujours de l'Etat de droit. Et de fait la référence à l'Etat de droit conserve une portée symbolique. Elle n'empêche sans doute pas une corruption majeure au sein de nombreuses institutions. Elle ne fait pas non 
plus obstacle à ce que des secteurs des forces armées commettent des violations évidentes des droits fondamentaux en pratiquant une guerre sale, en collusion fréquente avec les paramilitaires. Du moins, tous les acteurs en appellent à la restauration d'un vrai Etat de droit et de la paix et les confrontations s'accompagnent depuis vingt ans de processus presque constants de négociations comme si une rupture radicale était impensable. Le gouvernement actuel, on le sait, vient d'en entreprendre à son tour avec une grande audace.

4 La terreur colombienne actuelle ne met pas non plus en jeu des oppositions identitaires inventées ou héritées. Les haines ethniques, régionales, culturelles, n'y jouent pas de rôle. L'on ne détecte même pas un principe de division politique qui serait entériné par la majorité de la population.

5 La terreur est avant tout un outil au service des stratégies froidement calculées d'une grande variété de protagonistes. Elle est un sous-produit du mélange d'antagonisme, concurrence et coopération qui caractérise leurs interactions. Il se trouve seulement que c'est en frappant la population « civile » qu'ils cherchent à modifier les rapports de force entre eux.

6 Cet article a pour propos d'analyser plus avant ces expériences de la terreur.

7 Les rares travaux colombiens qui évoquent la terreur se situent sur deux plans séparés. Tantôt ils la décrivent comme l'une des composantes des stratégies de certains protagonistes - les paramilitaires principalement - tantôt ils la présentent à partir des épreuves ponctuelles racontées par les victimes mais sans les relier véritablement aux interactions entre les acteurs de la violence. J'entends au contraire lier étroitement les deux plans.

8 Les stratégies des protagonistes sont le plus souvent décrites sous trois aspects. En premier lieu, celui de la territorialisation, c'est-à-dire de l'imposition d'une règle autoritaire sur un espace donné. En second lieu, celui de la définition d'un temps orienté par leurs calculs et leurs objectifs. Finalement, celui de la construction d'allégeances ou de modes d'identification, volontaires ou inculqués de force.

9 C'est en retournant ces termes que je montrerai les formes de l'expérience de la terreur. Ce qui est territorialisation pour les protagonistes est vécu comme dématérialisation de l'espace par les victimes. Le temps maîtrisé des premiers devient éclatement des repères temporels pour les secondes. L'exigence d'identification suscite au contraire une dislocation des sujets manifestée dans les référents contradictoires auxquels ils se raccrochent.

10 Ce sont donc les expériences de dé-territorialisation, dé-temporalisation et désubjectivation à travers lesquelles je lirai l'empreinte négative des logiques stratégiques de terreur.

\section{La montée de la terreur}

11 Il y a encore quelque temps, tous les observateurs étaient cependant encore loin de prendre la dimension de cette terreur. Le terme était certes d'un usage fréquent. Mais il était surtout utilisé par les activistes liés à une organisation ou une autre pour stigmatiser les pratiques de l'adversaire. Certes, la population des grandes villes avait connu l'expérience de la terreur lorsque le Cartel de Medellin avait, de 1987 à 1993, recouru à des méthodes terroristes à grande échelle, à la fois ciblées et aveugles. Mais 
ce terrorisme, si meurtrier et intimidant qu'il fût, ne ruinait pas toute forme d'expression collective.

Il en va différemment désormais dans maintes zones qui ne sont pas seulement rurales mais comprennent aussi des périphéries urbaines. La terreur y est une réalité quotidienne qui affecte tous les habitants de ces zones et qui les enserre dans une peur permanente alimentée par les nombreux massacres, meurtres et enlèvements, mais aussi, tout simplement, par l'arbitraire que font peser les divers détenteurs organisés de la force.

Depuis les quartiers protégés des villes (en tout cas relativement protégés par rapport aux actions des protagonistes organisés de la violence), il a longtemps été difficile d'imaginer la situation des populations quotidiennement soumises à la vigilance des protagonistes armés et, moins encore, celle des populations de plus en plus nombreuses à être placées entre plusieurs feux. Chacun savait sans doute qu'une ville comme Barrancabermeja ${ }^{3}$ était divisée par des frontières invisibles et redoutables séparant les quartiers tenus par les diverses guérillas, les paramilitaires et les militaires, que de nombreuses bourgades rurales étaient disputées par les guérillas et les paramilitaires et qu'il en résultait de multiples massacres. Cela ne suffisait pas à faire de la terreur une donnée politique majeure et seul un massacre particulièrement important était susceptible d'émouvoir l'opinion.

14 Les déplacés avaient beau se multiplier à la lisière des villes, c'est à peine si leur existence était perçue. Il a fallu que leur nombre atteigne le million pour que l'Eglise et les ONG tournent leur regard vers le problème. Ce nombre approche maintenant 1800 000 , mais les autorités départementales et nationales continuent encore souvent à ignorer les problèmes majeurs qui en résultent. Ni l'opinion ni l'Etat ne pouvaient méconnaître les réalités quotidiennes de la violence mais il s'est longtemps agi d'en minimiser la gravité, les effets et, surtout, l'organisation. L'essentiel, disait-on, en était le résultat d'une criminalité petite et grande, quand il n'était pas mis sur le dos de "l'intolérance » et de la carence de l'esprit civique. Tout était fait pour " euphémiser » la dimension des confrontations dans lesquelles des armées diverses étaient impliquées. Du reste, le vocabulaire même traduisait ce souci général d'atténuer la gravité de la situation. Ce n'est pas un hasard si celui de "violence ", indéterminé et rassemblant les phénomènes les plus hétérogènes, était le seul à avoir cours. Parler de " guerre » ou de « guerre civile » était interdit comme le montrent l'indignation et les sarcasmes qui ont accompagné l'emploi du premier par le Président Virgilio Barco pour qualifier la lutte contre les narcoterroristes et par son successeur, Cesar Gaviria, pour évoquer une confrontation frontale avec les guérillas. Sans doute, les deux termes sont effectivement inadaptés, celui de guerre supposant une division «ami-ennemi» bien tranchée et celui de « guerre civile » impliquant une participation de la population qui précisément fait défaut puisque, dans son immense majorité, elle subit les affrontements et s'efforce d'échapper à l'emprise des acteurs.

15 Trois convictions, largement partagées et enracinées dans la tradition, expliquent ces attitudes. D'abord, celle selon laquelle il n'y a jamais eu dans l'histoire colombienne une séparation majeure entre l'univers institutionnel et celui régi par les normes de fait imposées par des réseaux détenant des ressources de pouvoir ou de force. Ensuite, celle selon laquelle il n'est pas de situations conflictuelles que des transactions ne puissent régler, le recours aux armes étant perçu comme une modalité, sinon normale, du moins acceptée de contester l'ordre institutionnel ${ }^{4}$. Finalement, celle selon laquelle «dire » 
les choses, et par exemple admettre l'aggravation constante de la violence, reviendrait à favoriser cette aggravation, voire à prendre parti en faveur d'une solution par la force.

Mais ces attitudes renvoient aussi aux particularités de la violence colombienne. Si elle ne se laisse pas ramener à un axe "ami-ennemi », c'est, on l'a dit, qu'elle est mise en œuvre par une multiplicité de protagonistes dont certains, comme les organisations de narcotrafiquants ou de nombreuses bandes urbaines, ont des intérêts propres et entretiennent avec les acteurs qui se proclament proprement politiques des relations fluctuantes, combinant coopération et antagonisme. Non seulement les conflits ne se sont pas étayés par des phénomènes identitaires mais ils sont loin de toujours coïncider avec des antagonismes sociaux stables, latents ou manifestes. Entre la violence organisée et la violence désorganisée partout présente existent des relations circulaires complexes, la première suscitant un contexte qui facilite le développement de la seconde, mais celle-ci en retour affectant les modalités de la première. Enfin l'Etat est loin d'avoir une stratégie unifiée : entre ses représentants nationaux, entre ceux-ci et ses représentants locaux, les dissensions s'étalent souvent au grand jour. Les forces armées sont elles-mêmes traversées par maintes tensions, et leurs rapports avec les forces policières sont loin d'être harmonieuses.

On sait que certains des protagonistes, les guérillas d'abord et les paramilitaires plus tard, ont progressivement concentré leurs efforts sur le contrôle de diverses ressources économiques, illégales comme la drogue, ou légales comme les activités minières et agricoles. C'est là un autre élément qui a pu favoriser leur ralliement aux méthodes de terreur. Ajoutés aux rançons, ces immenses revenus leur permettent désormais de développer leurs actions sans avoir à se soucier des sentiments de la population, de la soumettre sans avoir besoin de la convaincre.

Dans ces conditions, la violence paraît de plus en plus difficile à maîtriser. Le discrédit et l'impuissance du gouvernement Samper, l'étendue de la corruption, les revers enregistrés par les forces armées ${ }^{5}$ l'expansion des cultures de la drogue en dépit des mesures d'éradication : autant de facteurs qui ont contribué à son aggravation. Et les protagonistes armés recourent de plus en plus à la terreur comme à un instrument normal. Elle constitue une composante de leurs stratégies locales à mesure que paramilitaires et guérillas s'affrontent sans cesse davantage pour les mêmes territoires et que son emploi devient le moyen privilégié d'isoler l'adversaire en le coupant de la population. Des régions entières sont concernées. Aux régions du Moyen Magdalena, de l'Uraba et du Cordoba, s'ajoutent désormais notamment le nord-est de l'Antioquia, le Putumayo, certaines parties du Meta.

19 La nouveauté est cependant que la terreur affecte à présent des régions qui ne sont pas répertoriées comme théâtres directs de confrontations. Les groupes armés montent des opérations au plus loin de leurs fiefs, au moyen d'incursions rapides ou des assassinats sélectifs. Parfois les menaces et les rumeurs suffisent pour mettre les habitants aux abois. Quand les menaces sont mises à exécution, ces habitants découvrent que la protection promise par l'organisation installée parmi eux n'est qu'une vaine promesse. Les villes ne sont plus des refuges. Même si les principaux protagonistes armés n'y sont pas ouvertement installés, ils y entretiennent des réseaux clandestins ou y disposent de relais. Les déplacés en font l'expérience, qui s'aperçoivent vite au terme de leurs périples qu'ils retrouvent à leur arrivée les conditions qui prévalaient dans leur lieu d'origine et qu'ils sont voués soit à rester les otages des organisations dont ils 
dépendaient avant, soit à devenir des suspects s'ils passent sous la coupe d'organisations concurrentes ${ }^{6}$.

Tous les protagonistes n'usent pas également des mêmes méthodes de terreur. Les paramilitaires sont à l'évidence à l'origine de la grande majorité des massacres collectifs et, si l'on en croit les décomptes minutieux ${ }^{7}$, ils tuent au total plus que les guérillas. Mais, outre que les guérillas tuent déjà en nombre considérable et massacrent plus qu'occasionnellement, elles ont d'autres spécialités, depuis les enlèvements ---plus d'un millier leur sont imputables par an - jusqu'à toutes les formes d'intimidation, comme les « avertissements » aux maires et leur fréquente "punition », sans oublier le racket systématique et les sabotages à répétition des infrastructures pétrolières et électriques, qui ne sont guère moins génératrices de terreur. En pratiquant depuis quelque temps les enlèvements " au hasard » des barrages qu'elles établissent sur les routes, les fameuses "pêches miraculeuses ", et, comme au premier semestre de 1999, en procédant à la séquestration des passagers d'un avion de ligne détourné ou des assistants à une messe dans une église de $\mathrm{Cali}^{8}$, elles semblent même vouloir désormais étendre la terreur à l'ensemble du pays. Quant aux atrocités caractérisées, elles se distribuent assez bien entre tous les protagonistes.

21 L'ouverture de pourparlers préliminaires entre le gouvernement et les guérillas en juillet 1999 a certes pu susciter dans l'opinion l'espoir que de véritables négociations allaient s'engager et empêcher que la situation ne continue à se dégrader. Dix-huit mois après, les négociations n'ont toujours pas commencé avec l'ELN; tout juste entrouvertes avec les FARC, elles semblent ne devoir donner aucun résultat tangible avant longtemps; et le gouvernement n'a toujours pas défini le traitement qu'il entendait donner à la question paramilitaire ${ }^{9}$. Tout indique que chacun des protagonistes est bien davantage préoccupé de poursuivre les hostilités afin d'améliorer ses positions.

Dès lors, la terreur est susceptible de monter encore de plusieurs crans. Tout semble en effet montrer que les guérillas, les paramilitaires et certains secteurs militaires cherchent dorénavant à provoquer une polarisation générale du pays. Les FARC tendent à se présenter comme les seuls porte-parole de l'opposition au régime, et les paramilitaires comme les seuls porte-parole de l'opposition aux guérillas. Ceux qui ne sont pas d'un côté ou de l'autre deviennent ainsi la cible des uns ou des autres. On mentionnera plus loin le cas de la fragilité des "communautés de paix», ces regroupements destinés à soustraire la population aux pressions des acteurs armés. Mais cela vaut aussi pour les populations indiennes qui tentent vainement d'échapper aux coups et menaces des deux camps. Les assassinats non revendiqués qui ont frappé en 1999 des universitaires connus relèvent de la volonté de réduire au silence les quelques espaces indépendants subsistants. C'est l'idée même d'une "société civile » qui est mise en péril. A bien des égards, les protagonistes armés cherchent à accréditer l'idée qu'ils la représentent et qu'elle ne peut exister en dehors d'eux. L'ELN s'en proclame une émanation, les paramilitaires les gardiens, les FARC dénoncent l'inanité de la notion : autant de manières d'affirmer que, face à une " société civile en armes ", la société civile sans armes n'a pas de place.

La récession économique, d'une intensité sans précédent depuis 1929, qui touche actuellement la Colombie n'est pas de nature à atténuer la violence. Entraînant chômage et chute des salaires, secouant entreprises et institutions publiques, dévastant certaines régions, elle achève de déchirer le tissu social. Les guérillas peuvent avoir 
ainsi le sentiment que les "conditions objectives" vont venir à leur aide pour précipiter la polarisation qu'elles souhaitent.

\section{La dé-territorialisation}

\section{Les formes traditionnelles de territorialisation} Tous les acteurs armés n'ont pas une visée aussi claire de territorialisation. Les
militaires n'ont su que rarement mettre en œuvre des stratégies d'implantation solide us fréquemment procédé par des incursions momentanées, qui ne leur ttaient pas de remplir une fonction de protection permanente des populations, ni même d'acquérir une information fiable, ce qui se payait par un coût politique souvent élevé. Une guérilla comme le M19, passablement imprégnée d'une vision avant tout militariste, n'a pas non plus tenté d'établir des réseaux stables d'encadrement de la population : elle a quitté le Caqueta pour opérer dans d'autres départements, organisé des « campements populaires » à Medellin ou à Cali pendant le cessez-le-feu de 1984, pour les abandonner ensuite à leur propre sort, interféré parfois avec des mouvements sociaux mais s'est gardée d'établir avec eux des liens durables. L'on peut aussi douter que la notion de territorialisation s'applique aux narcotrafiquants. Ils ont sans doute disposé à divers moments d'une forte influence locale par le biais de leurs « contacts » avec les administrations (à Cali par exemple), de leurs réseaux armés propres (dans le Putumayo avec Gonzalo Rodriguez Gacha pendant un temps, dans le Moyen Magdalena avec le même et Pablo Escobar) ou de leur appui à des organisations paramilitaires plus vastes. Mais il n'était pas nécessairement de leur intérêt d'assurer la défense d'un espace précis s'ils voulaient se consacrer à l'essentiel, la bonne marche de leurs affaires, surtout une fois enclenché le conflit avec les autorités et avec les guérillas.

En revanche, des guérillas comme les FARC, l'ELN et l'EPL ${ }^{11}$ jusqu'à sa démobilisation et, par la suite, les paramilitaires et les milices ont bien visé à une telle territorialisation. 
Encore faut-il faire une distinction entre les uns et les autres selon que la territorialisation est à la base de leur existence ou qu'elle est seulement l'une des composantes de leurs stratégies. Les FARC se sont longtemps appuyées sur des zones du Tolima, du Huila et du Caqueta où des "autodéfenses paysannes " s'étaient formées lors des années de la Violencia . Elles ont ensuite élargi leur assise en organisant ce que l'on a appelé la " colonisation armée » de certaines régions ${ }^{12}$. De même, l'ELN continue à exercer son emprise sur les zones comme le Santander et l'est de l'Antioquia où elle a organisé ses premiers fronts. L'expansion rapide de ces deux organisations à partir des années 1980 et la multiplication de leurs fronts impliquent pourtant un autre type de territorialisation visant avant tout à la mainmise sur les pôles de production économique primaire. Comme l'a montré A. Reyes, la recherche d'une articulation avec les conflits agraires se fait plus incertaine ${ }^{13}$. S'ajoute depuis quelque temps un autre type de territorialisation reposant sur la mise sous tutelle des autorités locales et qui explique les multiples assassinats et enlèvements de maires et dirigeants municipaux par les FARC: l'ambition est cette fois de faire reconnaître une implantation quasiinstitutionnelle dans certaines zones, condition pour parvenir à un statut officiel de " partie belligérante ». Mais cette nouvelle territorialisation va de pair avec le primat d'une stratégie proprement militaire, manifeste dans les grandes offensives lancées en diverses occasions sur des régions dans lesquelles les guérillas n'ont pas de présence permanente. Il s'agit néanmoins encore d'une modalité de territorialisation, floue et mouvante, mais associée à la mise sur pied de réseaux d'intimidation et de surveillance. Les paramilitaires calquent leurs stratégies sur celles des guérillas. S'ils ne disposent pas de territoires historiques, ils sont parvenus à imposer leurs règles sur de vastes régions, comme le Cordoba et l'Uraba. Et, dans ces conditions, ils obtiennent également le ralliement d'une grande partie de la population, pas seulement des grands propriétaires fonciers, souvent narcotrafiquants reconvertis ${ }^{14}$, et des commerçants, mais aussi des simples paysans désireux de se soustraire à la violence. Eux aussi visent de plus à une territorialisation économique et politique. Plus encore que les guérillas, ils s'efforcent de s'assurer une territorialisation proprement militaire par l'établissement de têtes de pont jusqu'en pleine région ennemie.

30 La signification de la territorialisation est encore différente pour les milices et autres bandes urbaines : l'emprise sur le quartier est alors la condition même de consolidation de l'organisation et ce qui lui permet d'avoir accès à des ressources de pouvoir. Dans un même quartier, les organisations se métamorphosent ou se succèdent rapidement, les milices liées aux guérillas délogeant les sicarios, les groupes de délinquance organisée ou apolitiques prenant la place des milices, les groupes proches des paramilitaires remplaçant souvent à leur tour ces bandes. Subsiste la logique d'implantation de frontières assurant le monopole sur le quartier.

31 Les modalités d'emprise sur les populations sont encore plus variables que les types de territorialisation. L'exemple des zones de culture de la drogue, souvent décrit, constitue à bien des égards un cas particulier. Il ne fait pas de doute que les FARC ont pu longtemps s'y présenter en protectrices des colons et justifier ainsi les taxes prélevées : face à l'anomie qui prévalait souvent avant leur installation, la guérilla a instauré un ordre local, avec ses codes de justice, en même temps qu'elle défendait les colons contre les exactions des narcotrafiquants ou de l'armée. L'assentiment des colons à cette domination peut se justifier en termes de calcul rationnel - et l'on peut penser que les nombreux colons venus de la ville en espérant seulement accumuler 
aisément un magot et qui ne partagent donc pas la mémoire des hauts faits passés de la guérilla recourent effectivement à ce type de calcul. Il ne faut pas sous-estimer pour autant la part de la contrainte et les risques d'usure de l'autorité de la guérilla. Les grandes marches paysannes orchestrées par les FARC en 1996 pour protester contre les méthodes de fumigation des cultures manifestent certes le haut degré d'emprise de la guérilla. Il est peu probablequ'elles puissent se renouveler souvent. Le même modèle peut se retrouver ailleurs, mais sous des formes atténuées. Si la guérilla régente également la vie des habitants, elle peut avoir plus de mal à le faire entériner lorsque la tutelle n'est aucunement liée à des bénéfices économiques ou même à des bénéfices collectifs. Les exactions sont alors plus mal ressenties. Le cas de Puerto Boyaca est bien connu; à la fin des années 1970, la gauche révolutionnaire y détient le pouvoir municipal et la guérilla y est solidement implantée : mais les abus commis par un front des FARC vont faciliter le passage du municipe aux mains de groupes paramilitaires ${ }^{15}$. Même dans une bourgade comme Belen de Bajira ${ }^{16}$, où les FARC ont depuis le début organisé les colons, le poids de la contrainte et des sanctions compte autant que celui de la protection.

Cette modalité de territorialisation à travers l'emprise d'un réseau recourant à la contrainte n'est cependant pas inédite. Elle a été souvent mise en œuvre antérieurement par les partis traditionnels. Une belle monographie ${ }^{17}$ sur l'histoire de Trujillo, commune du Valle du Cauca, grosse productrice de café et située aux abords d'un des principaux axes routiers de communication, montre comment depuis 1930, les habitants ont été constamment soumis à de chefs locaux qui n'hésitaient pas à recourir aux assassinats et aux massacres pour imposer leur domination et étaient pourtant reconnus par les autorités départementales et nationales comme des intermédiaires tout à fait fréquentables. Bien d'autres communes appartenant à des régions centrales ont connu une histoire semblable, et plus encore celles appartenant à des périphéries géographiques. Dans le Meta, les leaders nationaux du libéralisme se sont longtemps appuyés sur d'anciens bandoleros pour encadrer la population. Dans l'Arauca, département devenu il y a quinze ans l'un des pôles de l'exploitation pétrolière et contrôlé par les guérillas, l'on a pu montrer que ces dernières mettaient en œuvre un clientélisme armé qui n'était pas si différent de celui précédemment exercé par les partis traditionnels ${ }^{18}$.

Il n'est donc pas surprenant que, notamment dans les régions de colonisation, les habitants aient pu considérer que la territorialisation sous l'égide d'un réseau armé ou d'un autre était dans l'ordre des choses. A la limite, elle pouvait passer pour un procédé nécessaire pour parvenir à un minimum d'intégration à la nation : en faisant surgir des entités spatialement définies et en suscitant des pouvoirs de fait, elle ouvrait la voie à une négociation avec les autorités centrales. Dans certaines parties de l'Uraba comme en Amazonie, les guérillas ont bien été à l'origine d'une plus grande attention du centre à l'égard des périphéries.

\section{La dé-territorialisation}

La terreur des dernières années a eu pour résultat de briser les relations de la population avec ces processus de territorialisation.

La concurrence entre des groupes armés pour le contrôle des mêmes espaces a déjà contribué à cette rupture. Les paramilitaires ont largement expulsé les guérillas de 
territoires comme le Cordoba et l'Uraba. La confrontation est en cours dans le Moyen Magdalena et dans d'autres régions. Elle traverse, on l'a dit, une ville comme Barrancabermeja et se manifeste dans les périphéries de métropoles comme Medellin où les milices sont fréquemment passées des «cruces $»^{19}$ avec les guérillas aux « cruces » avec les forces paramilitaires.

On pourrait penser qu'il s'agit seulement de la substitution des réseaux d'emprise et que la territorialisation se poursuit. Ce serait toutefois méconnaître les propriétés du nouveau contexte. L'incertitude est désormais une donnée majeure. Cela est évident dans les zones en cours de dispute. Dans le Sud du Cesar, nul ne peut encore savoir qui l'emportera, des guérillas ou des paramilitaires. Dans les périphéries urbaines, il n'est pas toujours aisé d'identifier le maître des lieux. Les bandes juvéniles ne cessent de changer d'orientations et les déplacés ne peuvent aisément connaitre les forces clandestines qui interviennent dans leurs campements. Même dans les régions «conquises» par un protagoniste, rien n'est assuré. Chassés aujourd'hui par les paramilitaires, les guérillas peuvent revenir demain. Elles s'y efforcent en ce moment, y compris dans l'Uraba et le Cordoba. Les frontières deviennent un peu partout indécises et fluctuantes.

Par ailleurs, les protagonistes armés administrent chaque jour la preuve qu'ils peuvent frapper au plus loin de leurs bases et jusqu'au cœur des fiefs de leurs adversaires. Les paramilitaires ont multiplié les actions dans des zones " historiques » des guérillas et les guérillas ont monté attentats et attaques dans les régions conquises par les paramilitaires. Là encore, les logiques classiques de territorialisation sont mises au défi.

Dans le même temps, les espaces soustraits à l'influence des acteurs armés se font de plus en plus rares. A l'initiative de l'Eglise, des "communautés de paix » ont été instituées dans certaines des zones les plus disputées afin de mettre la population à l'abri. Elles n'ont pas échappé aux coups des uns et des autres ${ }^{20}$. En d'autres cas, des bourgades et des zones indiennes ont proclamé leur "neutralité ", mais sans être davantage épargnées. Il n'est plus de lieu de refuge, et ceux qui fuient des zones les plus dangereuses s'exposent à retrouver à l'arrivée les menaces des mêmes acteurs.

Dans ces conditions, l'on peut parler d'une certaine homogénéisation de l'espace puisque tous ces points se trouvent exposés aux acteurs armés. Mais, surtout, cet espace se "dématérialise »: il devient un espace parcouru par des lignes virtuelles, celles liées aux circuits de menaces ou d'incursions. Chaque place devient seulement un point de convergence entre les réseaux par lesquels circule la force. Elle devient ainsi un «non-lieu ». Marc Augé a utilisé ce terme pour designer les espaces de circulation liés à la "surmodernité ${ }^{21}$. Je le reprends à mon compte, mais pour faire référence à des espaces qui, soustraits à leurs caractéristiques sociales, résultent des interactions entre des réseaux de force.

41 Même là où une certaine territorialisation se maintient, elle devient poreuse. Les habitants ont appris qu'il n'est pas de protection qui les garantisse contre l'irruption des adversaires, que leurs "protecteurs" actuels peuvent céder la place à leurs adversaires. Une bonne partie des paramilitaires se recrute parmi les anciens guérilleros ou miliciens. Les habitants savent donc que les guérilleros qui les encadrent aujourd'hui peuvent demain faire irruption sous l'uniforme des paramilitaires.

42 Le «non-lieu » est ainsi l'effet du pouvoir d'ubiquité dont disposent les organisations armées. Il n'y a plus d'espace privé dans lequel des liens sociaux puissent se construire. Chacun sait qu'il est potentiellement surveillé par tous les groupes armés. La confiance 
entre voisins est elle-même affectée : nombre d'entre eux ont des parents dans les deux camps et il n'est pas complètement exclu qu'ils puissent se transformer en « informateurs ». Les massacres des paramilitaires sont parfois perpétrés au hasard, souvent ils le sont sur la base de «listes» préétablies qui supposent de tels informateurs. Il arrive que ceux-ci assistent cagoulés à l'opération de sélection des victimes et les survivants ne doutent pas que, sous les cagoules, se dissimulent des voisins ou des proches.

Le "non-lieu " consiste également dans l'impossibilité de savoir comment l'on est catalogué à chaque instant par les réseaux armés. Leurs critères d'évaluation ne sont pas prévisibles. Sur un territoire encadré par un groupe armé, la population est organisée en cercles concentriques : à côté des membres à temps plein du groupe, il y a les membres à temps partiel, puis les collaborateurs occasionnels, puis les sympathisants, puis ceux qui rendent de menus services ou qui simplement assistent à des réunions, et naturellement ceux qui s'efforcent de se tenir à l'écart. Ce classement ne permet pas de préjuger les réactions possibles le jour venu du groupe "protecteur » et moins encore des groupes adverses. Se déplacer vers une bourgade voisine tenue par l'adversaire peut rendre suspect auprès du premier: nombreux sont les cas de personnes exécutées par les guérillas ou les paramilitaires simplement parce qu'en se rendant à un marché ou un lieu de travail proche ${ }^{22}$, elles ont franchi une frontière invisible et ont soudain été rangées parmi les sapos, les traîtres. A plus forte raison, lorsque les groupes adverses font une incursion sur le territoire, les habitants ignorent tout de la manière dont ils ont été à l'avance classés, « l'informateur " pouvant les avoir dénoncés comme collaborateurs actifs par simple désir de vengeance. Les litiges et traumatismes individuels issus d'années de violence ont une part non négligeable dans la sélection des victimes ${ }^{23}$. La participation même forcée à un mouvement collectif sous l'égide d'un groupe armé peut se payer cher. Nombreux sont ceux qui ont été tués ensuite parmi les participants aux gigantesques marches paysannes organisées dans le Caqueta en 1996 pour protester contre la politique de destruction des cultures de coca par fumigation, dirigeants mais aussi paysans de base.

Le «non-lieu » signifie le règne de la méfiance généralisée. Il n'est plus possible de se fier à l'organisation installée sur place, il devient nécessaire d'adopter une conduite de prudence avec tous. La loi du silence qui prévaut dans les zones de terreur n'est pas seulement imposée d'en haut. Elle gouverne les relations horizontales avec les semblables. C'est le tissu social qui se décompose.

Dans ces conditions, il n'est pas besoin d'en appeler à des théories sophistiquées du choix rationnel pour comprendre qu'il est de l'intérêt de chacun de chercher dans la mesure du possible à s'adapter individuellement aux contraintes qui pèsent sur lui et d'éviter de participer à toute action collective. Dans les zones de colonisation contrôlées par les guérillas, les formes d'organisation autonome, Juntas de Colonizadores ou Juntas comunales, qui réalisaient des travaux d'intérêt collectif, sont le plus souvent en sommeil ou instrumentalisées par les guérillas : y prendre part, c'est courir le risque d'être catalogué comme militant ou d'être recruté pour d'autres tâches. Parmi les déplacés, nombreux sont ceux qui font tout pour se fondre dans la population et échapper ainsi à l'étiquetage qui s'attache à leur condition.

Une telle fragilisation des solidarités, réduites souvent à quelques personnes très proches, et un tel repli sur les stratégies individuelles constituent d'autres modalités du «non-lieu ». 
La premiere concerne linteriorisation rémentaires.

La première concerne l'intériorisation des propriétés de l'espace à travers le sentiment de ne pouvoir se fixer en aucun endroit. Dans un climat de peur, les habitants des régions de violence sont nombreux à percevoir leur sort comme celui de migrants en
puissance. Ils disent n'être là que provisoirement. C'est vrai dans les zones disputées : ceux qui y demeurent encore évoquent souvent leur départ prochain. Le déplacement forcé est, bien sûr, la traduction la plus forte du non-lieu. Mais l'on peut aller plus loin. Même en d'autres régions, de nombreux habitants se considèrent comme étant simplement de passage. C'est par exemple le cas dans les régions de culture de la drogue, où nombre de colons se refusent, même quand ils ont accumulé quelques ressources, à améliorer leur habitat précaire en affirmant qu'ils entendent le plus tôt possible regagner la ville ou leur zone d'origine ${ }^{24}$. Il y a là une vision de la précarité de toute installation qui renvoie à une longue expérience historique. Le 20e siècle colombien est en effet marqué par l'occupation continue de territoires encore presque vides mais qui, une fois peuplés, se convertissent en foyers d'instabilité incitant fréquemment à des replis sur les villes. Plus encore, la mémoire de la Violencia des années 1950 alimente cette impression de précarité. Le souvenir des innombrables expulsions qui l'ont accompagnée demeure toujours aussi vif au point d'apparaître encore comme l'explication des trajectoires individuelles des décennies suivantes. A la question : "Pourquoi êtes-vous installés ici ?", nombre de colombiens répondent : "à cause de La Violencia ». C'est dire que violence et migrations sont vécues comme étroitement imbriquées. L'expérience actuelle de déplacement ranime ce souvenir, celui d'un destin de violence que l'on ne peut maîtriser. Mais sous une forme encore plus terrible puisqu'il n'est plus désormais de lieux sûrs de refuge.

La "dé-territorialisation » va par ailleurs de pair avec la dislocation des repères institutionnels. Aucun recours n'existe face à la violence. L'armée est souvent vue comme l'un de ses acteurs majeurs, aussi ou plus redoutable que les autres. La justice est paralysée et semble un appareil sans prise sur la réalité. Les partis politiques traditionnels se sont décomposés. Les maires, cibles privilégiées des protagonistes armés, sont placés dans les mêmes conditions que leurs administrés. Le scepticisme envers l'Etat n'a rien d'inédit : il est, lui aussi, ancré dans toute une tradition historique qui a toujours rendu incertaine la symbolique nationale. Son discrédit a pourtant rarement été aussi accentué et l'ampleur de la corruption de ces dernières années a contribué au sentiment de son irresponsabilité. Il n'y a rien pour faire barrage à la circulation des flux de force qui restructurent l'espace à leur manière. La déterritorialisation est l'expression concrète de la disparition de toute institutionnalité.

Enfin, un autre espace, non moins virtuel, intervient, celui lié à la globalisation. A certains égards, c'est certes un espace très concret. Il a des manifestations saisissables. A l'ouverture économique brutale pratiquée en 1991 est imputée le désastre dans lequel l'économie paysanne est plongée. Les ultimatums nord-américains imposent les fumigations qui obligent les colons à replanter ailleurs et les marchés étrangers déterminent les fluctuations des prix de la drogue. Les acteurs armés ont un pied dans les zones reculées, l'autre dans les réseaux internationaux de vente d'armes et de blanchiment d'argent. Le langage du droit est recouvert par la rhétorique internationale des droits de l'homme. N'importe quel colombien sait en appeler au "Droit international humanitaire » qui régit les situations de conflits internes. La Colombie était longtemps restée enfermée dans son provincialisme et son isolement, 
comme une sorte de "Tibet de l'Amérique du Sud» selon les termes d'un ancien Président. Voilà que s'établit un court-circuit entre le local et l'extérieur, qui achève de rompre les anciens espaces sociaux.

\section{La dé-temporalisation}

\section{Temps sociaux et temps stratégiques}

51 Les phénomènes de violence mettent en jeu de multiples temporalités. Elles se juxtaposent et se combinent selon des modalités instables.

52 De même que j'ai rappelé plus haut les différences sociales dans l'appréhension de l'espace, il faut rappeler ici la multiplicité des temps sociaux. Celui du paysan qui a hérité son lopin de terre n'est pas le même que le temps de celui qui s'est lancé dans la colonisation. Les aléas sont au centre de la perception du temps de ce dernier ainsi qu'Alfredo Molano l'a fort bien suggéré en racontant les trajectoires à rebondissements de colons qui perdent et retrouvent leur famille, défrichent leur terre pour s'en trouver ensuite dépossédés, voient leur travail ruiné par les inondations et autres calamités naturelles ${ }^{25}$. L'appréhension du temps des jeunes urbains est encore différente et varie selon les époques. Alonso Salazar ${ }^{26}$ et Fernando Vallejo ${ }^{27}$ ont décrit le temps des sicarios, ce temps bref et sans futur, marqué par la succession des meurtres, des défis et des confrontations avec la mort. Les bandes juvéniles actuelles, celles qui prétendent assurer la survie de leurs quartiers, ne se réfèrent plus à un temps aussi court. L'expérience du déplacement s'accompagne d'une altération des repères temporels dans la mesure où elle implique une attente sur laquelle le déplacé n'a plus guère de prise. Toutes ces temporalités laissent des traces dans celles de la violence, car celle-ci n'opère pas dans un vide social.

53 Les temps sociaux influent sur les modes de l'action collective. L'on peut détecter des moments forts dans lesquels, à travers la participation à une telle action, des populations ont l'impression de mettre en scène leurs caractéristiques sociales, lors d'invasions de terres ou lors de marches par exemple. Ils impriment aussi leurs marques sur les acteurs, y compris les acteurs armés. Les FARC des années 60 sont encore largement en prise avec l'espace et le temps paysans. La localité ou la région constituent souvent leur horizon spatial, le temps des récoltes ou de mise en valeur des terres, leur horizon temporel. Le fait de pouvoir compter sur la durée, sans céder à l'impatience, est au cœur de l'histoire de cette guérilla cinquantenaire. Les témoignages récemment publiés à propos de l'histoire de l'ELN et de l'EPL laissent au contraire apparaître combien le temps des cadres d'origine urbaine, inspiré de la théorie politique, se combine difficilement avec le temps paysan.

Ces différences se répercutent aussi sur les temps stratégiques, ceux qui président aux plans de confrontation ou de négociation. Les gouvernements ont quatre ans pour agir, et même moins puisqu'ils perdent généralement leur capacité d'initiative au bout de deux ou trois ans. Le savoir accumulé par l'un ne se transmet guère au suivant, tous donnant le sentiment de vouloir tout reprendre à zéro et le personnel dirigeant se renouvelant à toute allure. Les guérillas, qui se targuent de leur quarante années d'existence, ont au contraire appris l'importance de compter sur un temps long. Les autres acteurs illégaux échappent également à la périodisation rapide de la vie politique. Il en résulte une asymétrie fondamentale, sensible dans la conduite des 
opérations armées mais plus encore dans celle des négociations. Les faire s'éterniser est pour les guérillas un moyen facile de fragiliser tout gouvernement.

La maîtrise du temps n'est pas qu'une affaire de stratégie. Elle passe aussi par l'aptitude à se réclamer des temps sociaux de la population. Là encore, les guérillas et autres acteurs illégaux ont longtemps eu un avantage sur les autorités gouvernementales. En prétendant continuer à prendre en charge les humiliations héritées de la Violencia ou une «culture de l'illégalité » née de la nécessité ressentie de résister aux injustices du régime, ils ont réussi à faire croire qu'ils étaient porteurs d'une vision de l'avenir. C'est ce que la terreur remet en question en provoquant une disjonction radicale entre les temps sociaux et le temps qui dérive des stratégies de confrontation.

\section{Le temps de l'événement et le temps mythique}

56 La problématique est en effet tout autre pour la population exposée à la terreur. La marque des temps sociaux est, certes, toujours sensible. Mais le temps n'est plus nécessairement orienté. Il est bien davantage pris à la fois dans la succession événementielle et dans une représentation mythique de l'éternelle répétition.

«Ce jour-là, ils ont fait irruption » : cette phrase si souvent formulée par les déplacés indique bien l'aspect événementiel de la catastrophe. L'événement pouvait être attendu et redouté, et bien des assassinats ou des menaces avaient pu le précéder. Il ne se produit pas moins comme une surprise quand il touche le sort personnel et produit une coupure brutale, un avant et un après. Il en va de même à un niveau plus général. Les journaux font chaque jour des mentions discrètes des assassinats qui se déroulent en diverses parties du territoire. Cette actualité n'émeut guère, elle est devenue une routine. Que surviennent le massacre d'un nombre exceptionnellement élevé de personnes ou l'assassinat d'une personnalité de premier plan, Jaime Pardo Leal, Bernardo Jaramillo, Luis Carlos Galan, Carlos Pizarro, Alvaro Gomez Hurtado ou Jaime Garzon $^{28}$, l'émotion envahit un instant la presse et l'opinion comme si un tabou avait été brisé, et une cassure était intervenue. Les événements exceptionnels s'insèrent cependant vite dans une routine, l'un chassant l'autre. La preuve en est que leur mémoire se perd rapidement. Aucun d'entre eux n'a valeur de commencement et tous finissent par se confondre en s'accumulant. Chacun laisse seulement une trace, un peu comme la queue d'une comète, mais une trace qui ne s'insère pas dans une histoire structurée. D'autant que, dans la majorité des cas, un doute subsiste sur les auteurs des crimes, laissant le champ libre aux rumeurs les plus contradictoires. Banalité de la violence et exceptionnalité de l'événement ont tôt fait de se mêler dans une trame imprécise. Le déroulement ininterrompu des assassinats fait que les repères s'effacent et que l'oubli devienne constitutif du rapport à la réalité. Le " présentéisme ${ }^{29}$ prévaut, qui revient à s'inscrire dans un temps privé d'« horizon d'attente» aussi bien que de jalons fixes dans le passé.

La terreur qui sévit à présent renforce encore ce "présentéisme » et instaure une disjonction entre un temps purement événementiel et un temps mythique figé, celui de la répétition.

La répétition est la catégorie à travers laquelle les événements sont appréhendés. L'immense majorité des Colombiens reste convaincue que les drames d'aujourd'hui sont identiques à ceux de La Violencia et que ceux de La Violencia l'étaient à ceux des guerres civiles du 19e siècle. La répétition suggère bien qu'une temporalité mythique 
sert aussi de référence, selon laquelle la même violence est là « depuis toujours » et est vouée à se reproduire sans fin.

Cette temporalité n'est en fait que l'un des aspects d'une représentation mythique plus globale. Car la violence apparaît souvent comme la face visible d'une réalité souterraine, comparable à un flux de forces antagonistes ou à une catastrophe naturelle, et qui gouverne les hommes malgré eux. Il a souvent été relevé que, dans les années 1950, la violence prenait, dans les déclarations des victimes, l'aspect d'une sorte de démiurge qui, plus encore que les protagonistes identifiables, était responsable de la calamité. Dans le langage courant, c'était «La Violencia » qui avait perpétré telle ou telle horreur. Il n'en va pas toujours différemment aujourd'hui. Le « ils » de « ils ont fait irruption ", témoigne que, même si le responsable est connu, il est vain de le nommer parce qu'il n'est qu'un avatar d'une adversité sans visage.

De la structure mythique relève aussi la tendance à attribuer la responsabilité de la violence à des traits enracinés dans la "nature » des Colombiens. Comme les jaguars et les serpents des mythes canoniques, ces derniers seraient portés par cette "nature » à agir comme ils le font, à enfreindre les lois, manquer à leur parole, refuser de coopérer entre eux, recourir à la violence en guise d'argument. Les stéréotypes concernant je ne sais quelle «culture de l'intolérance» ou "psychologie collective» ne sont pas seulement présents dans une sorte de discours tout fait, mais repris dans bien des analyses savantes. On peut sans doute discerner dans cette pratique de l'autodénigrement la marque de l'imprégnation par une tradition catholique, prompte à dénoncer la culpabilité collective et à délaisser les responsabilités spécifiques ${ }^{30}$. Mais il faut aussi y voir l'impossibilité de mettre les événements en récit.

Entre l'événement et le mythe, le passé du présent et le présent du passé, les populations soumises à la violence actuelle oscillent en permanence. Ce qui leur fait défaut est la possibilité d'être partie prenante à une histoire orientée vers l'avenir. En ce sens, le rapport au temps est à l'opposé de celui qui règne par exemple au Brésil où le présent incertain n'empêche jamais l'avenir d'être certain.

\section{De la violence sans histoire à sa représentation kaléidoscopique}

63 Selon la formule fameuse de Freud, l'inconscient n'a pas d'histoire. L'on pourrait appliquer la remarque à la violence. Entre l'événement et le mythe, un maillon manque, qui est celui d'une histoire instituée et instituante.

Si l'histoire de la Colombie depuis l'Indépendance se lit si fréquemment sous le signe de la violence alors même que celle-ci revêt dans certaines phases une ampleur qui n'est guère différente de celle existant dans d'autres pays ${ }^{31}$, c'est d'abord qu'aucun point de vue unifié ne se consolide. Pendant cent cinquante ans, la concurrence entre les deux partis et entre les deux sous-cultures antagonistes qui la prolonge produit deux déchiffrements distincts de cette histoire.

Dès lors, en place d'événements symboliques qui seraient reconnus comme fondateurs d'une communauté nationale, seuls des événements catastrophiques subsistent dans la mémoire, qui attestent du déchirement inéluctable de la société. Tel est le cas de l'assassinat de Gaitan, le 9 avril 1948 et du soulèvement qui a suivi. Sans doute est-ce l'une des seules dates largement remémorées, mais parce qu'elle est celle d'un fait historique qui réactive le sentiment d'une division irréductible et qui est perçue 
comme le point de départ de tous les soubresauts qui se sont ensuite succédé jusqu'à la violence actuelle.

La plupart des pays ont sans doute connu des catastrophes comparables qui, sur le moment, ont fait vaciller les repères communs. Mais dirigeants politiques et historiens se sont attelés après coup, dans un hégélianisme inconscient, à intégrer le « moment du négatif» dans une synthèse qui est le propre des « récits nationaux ». Il n'en va pas de même en Colombie. Le 9 avril demeure toujours comme une plaie non cicatrisée qui sert seulement à justifier les thèses des uns ou des autres, des libéraux ou des conservateurs, des partisans de l'ordre ou de ceux de la révolution. Lorsqu'elles ont formé en 1958 le Front National qui scellait la réconciliation des deux partis traditionnels, les élites politiques se sont entendues pour jeter un voile d'oubli sur le passé et mettre en accusation les quelques historiens qui voulaient l'analyser. Les élites morales, c'est-à-dire d'abord l'Eglise, leur ont prêté le concours de leur autorité, car elles ne désiraient pas avoir à réfléchir sur leur propre responsabilité dans ce passé. Il n'est donc pas surprenant que, lorsque les circonstances obligent à y revenir, s'imposent à nouveau les commentaires les plus divergents comme si la plaie était toujours ouverte : on a pu le constater en 1998, lors du cinquantenaire du Bogotazo, où la presse a fait assaut de déplorations sur la barbarie des masses ou celle des anciens adversaires politiques. Il n'est pas étonnant non plus que, faute de récits institutionnels ou savants, une mémoire sauvage et individualisée continue à se manifester un demisiècle après. N'importe quel jeune guérillero ou tueur urbain justifiera son comportement par les traces laissées dans sa famille par cet épisode, voire par les séquelles dont il est personnellement porteur.

L'absence de repères autres que catastrophiques tient aussi au style de l'action publique. Fait de transactions et de mesures au jour le jour, soustrait aux pressions populistes ou nationalistes, il n'a préservé une stabilité institutionnelle qu'en se gardant de transformations qui auraient introduit une rupture au moins relative: il n'est ni réforme agraire, ni altération de l'Etat, ni avènement d'un parti nouveau pour donner l'impression d'une mutation en profondeur. Les seules cassures sont négatives. La "précarité de l'Etat ", au delà de ses aspects techniques, renvoie à sa défaillance pour provoquer des inflexions qui créeraient des repères d'une autre nature et susciteraient un sens de l'appartenance commune à la nation.

La terreur ne fait qu'exacerber cette carence. Ni l'Etat ni les organisations de la société civile ne parviennent à en proposer un déchiffrement. L'Eglise n'ose pas en appeler à des principes éthiques et préfère se mêler aux transactions entre tous les acteurs. S'impose la représentation d'une guerre de tous contre tous.

Dès lors, le mélange de l'événementiel et du mythique préside à une appréhension de l'histoire actuelle en termes que j'ai appelés ailleurs « kaléidoscopiques »32.

Par là, je n'entends pas seulement souligner la difficulté d'inscrire les événements dans une trame d'ensemble. J'entends suggérer d'autres phénomènes.

71 D'abord, la vision même des « causes » et des particularités de la violence ne cesse de se modifier au gré de ces événements. Au début des années 1980 prévalait une lecture politique selon laquelle les blocages du Front National portaient la responsabilité de la dérive. Elle est maintenant remise au goût du jour par les guérillas avec l'accusation de «terrorisme d'Etat». L'anomie sociale a été ensuite évoquée et un livre de grand retentissement a prétendu établir que la "violence de tous les jours » était autrement plus importante que celle mise en œuvre par les protagonistes armés ${ }^{33}$. Puis, selon les 
conjonctures, le narcotrafic, le néolibéralisme ou le grand capital ont à leur tour soustendu les explications. "L'intervention américaine" est en train de déplacer ces analyses. La validité de ces facteurs n'est pas ici ce qui est en question. Ce qui l'est, c'est qu'ils se substituent les uns aux autres, dans le sens commun comme dans les analyses savantes, au rythme des événements et que le dernier en date de ceux-ci soit susceptible de faire vaciller tous les schémas antérieurs.

Ensuite, l'opinion est vouée à fluctuer sans cesse sans prendre en considération les dynamiques de longue durée qui sous-tendent la violence. La mort de Pablo Escobar lui fait croire que le problème de la drogue prend fin. L'ouverture de négociations avec les guérillas entraîne une euphorie qui retombe dès qu'il devient évident que, négociations ou pas, la confrontation est amenée à se prolonger pendant des années. De telles oscillations, en même temps qu'elles manifestent l'incapacité des gouvernements à dessiner des perspectives à long terme, affaiblissent leurs moyens d'action en leur enlevant toute crédibilité aussitôt qu'ils doivent reconnaître les innombrables obstacles à la paix.

Enfin, dans les zones où la terreur sévit, la population est plus encore privée des repères temporels qui lui permettraient d'imaginer un au delà du moment présent. La vision kaléidoscopique ne relève plus des simples représentations : elle est celle qui se déduit des faits bruts qui se déroulent et qui désarticulent le lien social. Chacun n'a plus que des bribes d'histoire personnelle, séparées par des coupures et dépourvues de tout « horizon d'attente ». Telle est la forme la plus profonde de la « dé-temporalisation ».

\section{La dé-subjectivation}

Par ce terme, j'entends suggérer l'homologie avec les raisonnements antérieurs. Il est bien sûr discutable. L'individu soumis à la terreur continue au moins à s'affirmer comme sujet par le biais d'une identité narrative ${ }^{34}$. Peut-être aussi par le sentiment de "l'espèce humaine ${ }^{35}$ qu'il découvre en lui-même. Il n'en reste pas moins qu'il est arraché à sa trame sociale, selon le mot de $\mathrm{H}$. Arendt à propos des apatrides ${ }^{36}$. J'entends surtout montrer que l'épreuve de la terreur, en rendant impossible l'insertion du récit individuel dans une histoire collective, peut donner à l'identité narrative elle-même un caractère discontinu. Et que, soumis à des menaces multiples, l'individu s'installe dans une pluralité de références normatives apparemment contradictoires, comme s'il ne parvenait plus à opérer un choix personnel entre ce qui lui deviendrait totalement extérieur. La "dé-subjectivation» n'est rien d'autre et rien de moins que cet éclatement du sujet.

\section{L'organisation violente et l'imposition identitaire}

Tant que la violence est perçue comme banale, l'intervention des protagonistes armés peut, en imposant des allégeances, contribuer néanmoins à la construction individuelle et collective de sujets.

Il en va ainsi lorsque les réseaux d'emprise sont perçus comme imposant une «loi » commune qui protège. Qu'il soit possible d'évoquer la formation du sujet individuel par l'intermédiaire de la contrainte, maints témoignages d'adolescents entrés dans les guérillas ou dans les bandes de quartier tendent à le prouver. S'il existe un recrutement forcé, l'adhésion est le plus souvent plus ou moins choisie. Et, si ces jeunes la justifient 
souvent, sans besoin d'arguments politiques, par la séduction des armes ou de l'uniforme, il convient de relever que ces signes extérieurs constituent une manière de se soustraire aux aléas du monde ordinaire et d'accéder au statut associé à l'appartenance à une organisation. L'adhésion est aussi la condition pour être protégé par un système d'autorité. Les défaillances de l'autorité tant paternelle qu'institutionnelle concourent-elles à cette demande d'autorité ? On peut en débattre. Mais on ne peut douter que la relation avec un système d'autorité contribue à la consolidation de l'image de soi. Ce n'est pas un hasard si ceux qui ont déposé les armes font état d'un désarroi, voire de leur sentiment de déchéance.

identité ainsi construite a ses limites. J'ai parlé de choix mais celui-ci est, au moins partiellement, arbitraire. Il est fréquemment le produit de l'offre locale en groupes armés ou bien encore de l'attrait exercé par celui de ces groupes qui semble avoir le vent en poupe. Il n'est pas rare que, dans une même famille, les enfants rejoignent des organisations opposées. C'est dire qu'une même sociabilité originaire s'accommode d'une diversité d'options. Dans la mesure où l'insertion organisationnelle compte plus que les convictions ou les idéologies, il n'est pas étonnant que les affiliations puissent changer au fil du temps. Les profils de carrière sont ouverts et variés. Dans les années 1980, les organisations délinquantes de Medellin étaient hiérarchisées en fonction de leur proximité avec le cartel de Pablo Escobar et la réussite consistait à être embauché par celles travaillant directement pour lui. D'autres trajectoires consistent à passer des bandes de quartier aux guérillas ou aux paramilitaires. Certaines, en sens inverse, conduisent à transiter de ces organisations politiques vers la délinquance. Il y a enfin celles caractérisées par le ralliement aux organisations politiques opposées. Plus qu'à des identités stables, il convient de se référer à des identifications mouvantes.

Cette fluidité vient aussi de ce que les organisations ont renoncé depuis longtemps à un discours de persuasion. Même les guérillas ne prennent plus guère le temps de donner une formation politique aux recrues, en dehors de quelques formules rudimentaires ${ }^{37}$, ni d'élaborer de belles proclamations à l'adresse de l'opinion publique. Paradoxalement, les paramilitaires sont en train de mettre à profit ce vide pour montrer qu'ils sont capables d'être plus "théoriciens" que leurs adversaires en rédigeant, avec le concours d'«intellectuels" sympathisants, un argumentaire justificatif. Il reste que la guerre se mène maintenant en actes et non en paroles. Ce sont les massacres et les assassinats qui sont chargés de "parler ». Ils marquent les frontières, disent les objectifs, expriment les normes, tiennent lieu de sens ${ }^{38}$. A la fameuse formule du linguiste Austin à propos des performatifs, selon laquelle «dire, c'est faire ", s'oppose une réalité dans laquelle « faire, c'est dire ».

Du reste, même quand ces actes prennent l'aspect d'atrocités, ils ne s'accompagnent que rarement d'une mise en scène ritualisée de l'horreur. Lors de la Violencia, les viols étaient souvent systématiques et les bandes avaient coutume de profaner les corps de manière codifiée afin de mieux manifester la négation de l'autre. Si les cas sont moins fréquents aujourd'hui, ce n'est pas seulement parce que l'arme à feu a remplacé la machette et que la quantité tient lieu de code au lieu de la marque visible de la cruauté, c'est aussi que l'usage de la terreur ne se fonde plus sur une division politique de la société qui lui préexisterait, mais sert à imposer une division largement arbitraire renvoyant aux stratégies territoriales. Elle ne met pas en jeu du sacré, elle reste instrumentale. 
80 L'identité des victimes comme celle des agents en résulte beaucoup plus floue. Les premières n'ont pas toujours des affiliations définies. Les seconds se conduisent en fonction de routines et seule la poursuite de celles-ci assurent leur identité. L'on ne dispose pas d'étude systématique sur les nombreux « retraités » de l'action armée. Bien des indications suggèrent que, passé le moment du premier désarroi, ils s'insèrent dans la société comme s'ils n'avaient participé à rien qui eut une signification pour eux.

\section{La trajectoire personnelle comme récit non inséré dans une histoire collective}

81 Ceux qui sont soumis à la terreur racontent le plus souvent leur vie sous la forme de la description du passage d'un lieu à un autre. La violence qui les a fait partir d'un endroit les a rattrapés dans un autre. Entre les épisodes, il y a souvent des vides, qui sont comme des indices d'une discontinuité qui les affecte dans leur permanence comme sujets. Parfois, ils semblent n'être plus les mêmes aux diverses étapes: paysans traditionnels, colons plus ou moins chanceux, mis en tutelle par un groupe armé, déplacés - autant d'avatars qui comportent une redéfinition de soi. Sans compter les assassinats de proches et les menaces du présent. L'identité propre se transforme dans le temps.

82 La mise en récit de la trajectoire personnelle ne va donc déjà pas de soi. Les fragments successifs dans lesquels elle s'exprime ne se raccordent pas. A certains instants, les sujets sont agissants, à d'autres, ils sont seulement « agis ». Si l'énumération des lieux, même devenus des non-lieux, sert de trame, c'est qu'ils peuvent être nommés tandis que la source des souffrances peut rarement l'être.

83 A plus forte raison, l'inscription des récits personnels dans un récit collectif ne se produit qu'exceptionnellement. Cela supposerait soit la possibilité d'assumer un point de vue en surplomb, soit l'adhésion déclarée à la cause de l'un des protagonistes. Or les victimes préfèrent le plus souvent assumer le fait qu'elles parlent à partir d'une perspective dont elles ne peuvent rendre compte. Ce qui revient à suggérer qu'elles ignorent quelle est l'histoire collective qui se joue et même s'il s'en joue véritablement une.

\section{Les référents contradictoires du sujet}

84 La difficulté du sujet à rassembler ses expériences dans une trame pourvue de sens se manifeste d'une autre manière: les références normatives opposées à quoi il recourt dès qu'il prétend porter un jugement sur sa situation ou sur la situation d'ensemble.

Là encore les entretiens tant avec les déplacés qu'avec des habitants des zones disputées mettent vite en lumière cet "éclectisme». La nostalgie de «la moralité d'autrefois » fait bon ménage avec l'admiration pour les narcotrafiquants. L'appel au respect des lois se combine avec l'acceptation de toutes formes d'illégalité. Le mépris envers la politique s'associe avec l'intérêt pour les élections ${ }^{39}$. La valorisation de l'action collective coexiste avec la revendication du «chacun pour soi ». L'honnêteté est prônée mais le "rebusque ", art de tourner les obstacles un peu par n'importe quel moyen, est érigé en règle. 
Ces référents ne sont peut-être contradictoires qu'en apparence. Ils sont profondément compatibles du point de vue de celui qui les énonce comme si, simultanément ou tour à tour, leur utilisation s'imposait pour faire face aux problèmes qui se posent. Dans un ouvrage récent sur un quartier de Bogota, un sociologue a bien décrit comment la défiance sous-tendait les accords et comment l'appel à la communauté allait de pair avec la violation de toutes les normes communautaires ${ }^{40}$. Les comportements changeaient en fait selon les nécessités du moment.

« Dé-subjectivation »? Il peut en effet arriver dans des moments extrêmes que le sujet ne parvienne plus à assumer en première personne ce qu'il subit ou fait. Mais il est plus exact de parler de subjectivité éclatée. La division du sujet entre des référents opposés ou des registres de vie superposés n'a, elle, rien d'exceptionnel. Aux côtés de la déterritorialisation et de l'éclatement des repères temporels, elle est une autre manifestation de la terreur. Celle qui se manifeste dans le récit des déplacés par la mention du moment où il n'a plus eu la possibilité de s'adapter aux circonstances, où il en devient « le jouet » pour reprendre une expression commune.

B8 Je me suis efforcé au long de ces pages de rendre compte des formes de la terreur. Ces formes sont à la fois objectives et subjectives. Même le territoire et le non-lieu appartiennent aux deux registres. Ils délimitent l'horizon de la vie présente mais leurs frontières sont largement immatérielles, surtout quand elles sont définies par la confluence des menaces. Le temps ne cesse pas d'être ancré dans les expériences sociales alors même qu'il se fragmente au gré des événements ou prend une consistance mythique. Quant au sujet, il conserve au moins la consistance liée à la possibilité de faire le récit de ses épreuves même s'il est confronté par ailleurs à l'éclatement des référents sociaux.

9 La terreur implique en fait le brouillage de la distinction entre l'objectif et le subjectif, des conditions concrètes et des représentations. Toute situation est évaluée à l'aune de " ce qui peut arriver ", tout événement semble après coup " avoir été annoncé », tout lieu est destiné à être perdu.

- En revanche, et c'est fondamental, elle détruit tout imaginaire, si l'on entend par ce terme la faculté de se rapporter à une signification unificatrice. La terreur est en fait ressentie comme un enchevêtrement de faits, de calculs prosaïques, de souffrances. Cette carence d'imaginaire influe jusque sur la perception du politique. Celui-ci ne semble plus lui même composé que de relations de force ou de transactions utilitaires. De sa fonction instituante, il ne subsiste rien, surtout dans les régions de violence.

91 J'ai mentionné au départ que guérillas et paramilitaires s'efforçaient à présent de susciter la polarisation de la société. Malgré l'usage de la terreur, ils n'y sont pas parvenus. Certes ils peuvent tenter d'imposer le silence là où ils ne font pas régner la soumission. Mais ils ne réussissent pas à faire croire qu'une telle polarisation serait porteuse d'un avenir, et moins encore d'un avenir qui chanterait.

2 Dans les zones les plus affectées par la terreur, la dislocation des liens sociaux habituels interdit à l'évidence toute réaction collective autonome et ouvre la voie à l'adoption des critères de jugement contradictoires. Mais il faut ajouter une nuance importante à ce constat. Le retrait sur soi et l'oscillation normative sont une façon d'indiquer également la distance à l'égard de tous les protagonistes de la violence. Le «ils » utilisé pour les désigner suggère en creux un "nous ", l'ensemble de ceux qui subissent les conséquences de leurs actions. Un « nous» qui ne peut s'organiser mais qui atteste au 
moins, par delà l'apparente soumission, du maintien d'un quant à soi qui va à l'encontre de la polarisation.

$93 \mathrm{Au}$ plan national, l'opinion publique fait d'ailleurs de plus en plus irruption pour protester contre la violence. Les grandes manifestations en faveur de la paix qui ont eu lieu en 1999 en apportent la preuve. Il reste qu'elles demeurent encore discontinues, comme prises elles-mêmes dans une durée kaléidoscopique, et que les porte-parole de la « société civile » sont incapables de leur conférer une architecture permanente.

Face à la désinstitutionnalisation engendrée par la violence, le défi est bien de recréer de l'institutionnalité. C'est la condition pour susciter à nouveau des repères partagés. Le «nous" pourrait alors prendre une forme concrète et les manifestations se transformer en un phénomène politique susceptible de modifier le contexte de la violence. Il est probable que ce changement n'est possible qu'en prenant appui sur des mécanismes de médiation internationale, qui ne consistent pas en une immixtion directe, mais en une contribution à la recréation d'une sphère publique élargie. Peutêtre n'est-il pas de tâche plus urgente pour le gouvernement et les porte-parole de la société civile s'ils veulent éviter la généralisation de la terreur.

\section{NOTES}

1. «De la banalité de la violence à la terreur : le cas colombien ", Cultures \& Conflits, $n^{\circ} 24 / 25$, Hiver-Printemps 1997, pp.159-193.

2. Cf. J.A. Bejarano, «Democracia, conflicto y eficiencia économica », in Construir la Paz, Bogota, CEREC, 1990.

3. Ville de 300000 habitants au centre de la Colombie où se trouve la plus grande raffinerie de pétrole et qui possède une longue tradition de luttes ouvrières.

4. Au point d'être admis jusqu'à tout récemment dans les textes légaux puisque la rébellion politique n'était punissable que par des peines très légères. Il y a là une survivance du $19 \mathrm{e}$ siècle où, guerre civile et politique étant largement confondues, il convenait de laisser ouverte la porte à la réintégration des rebelles.

5. Ces revers ont commencé en 1995 avec la prise par les FARC d'une importante base militaire du département du Caqueta et la prise d'une soixantaine de "prisonniers ». Ils se sont ensuite succédé jusqu'en 1998, les FARC détenant alors au total près de 300 militaires ou policiers. Elles en ont désormais plus de 500Cette accumulation de "prisonniers " semble destinée à obtenir le moment venu le statut international de «partie belligérante» et pose un grave problème au gouvernement.

6. Cf. mon analyse de la problématique des déplacés dans l'article « La perdida de los derechos, del significado de la experiencia y de la insercion social: A proposito de los desplazados en Colombia ", Estudios Politicos, Medellin, Université d'Antioquia, n 14, janvier-juin 1999, pp.13-30.

7. Les données les plus fiables sont celles qui sont présentées par Camilo Echandia dans diverses études. En français, l'on peut se reporter à deux de ses articles publiés dans les numéros 16 (1995) et 34 (1999) de la revue Problèmes d'Amérique Latine.

8. L'ELN est responsable de ces deux dernières actions, les FARC de la plupart des "pêches miraculeuses »Il est à noter que, si les deux actions de l'ELN ont d'abord semblé destinées à 
obliger le gouvernement à entériner plus rapidement l'ouverture de négociations, elles ont donné lieu à des demandes de rançons et certaines des victimes sont encore retenues faute de s'en être acquittées.

9. Les pourparlers préliminaires avec les FARC ont duré quinze mois, malgré les nombreuses concessions faites par le gouvernement. Les négociations proprement dites n'ont commencé qu'en octobre 1999, en fait en janvier 2000. La manière dont leur agenda a été fixé implique qu'elles ne vont pas toucher les vraies questions dans un délai prévisible. Sur la première année de pourparlers, cf. D. Pécaut, "Colombie : une paix insaisissable », Problèmes d'Amérique Latine, $\mathrm{n}^{\circ}$ 34, juillet-septembre 1999, pp.5-32.

10. El hombre y la tierra en Boyaca. Desarrollo historico de una sociedad minifundista, Bogota, 2e éd., 1973.

11. Rappelons que l'EPL, surtout implanté dans le Cordoba et l'Uraba, a déposé les armes en 1991, peu après le M19. Un groupe, dirigé par Francisco Caraballo, a cependant continué une lutte qui a pris de plus en plus l'aspect d'un pur banditisme. En 1999, les FARC en ont du reste exterminé certains membres qui, à Barrancabermeja, se livraient à la délinquance. Une grande partie de ceux qui ont déposé les armes ont été pourchassés par les FARC, ce qui les a obligés à les reprendre, mais cette fois aux côtés des paramilitaires.

12. Pour reprendre l'expression créée par William Ramirez Tobon.

13. "Conflictos y territorio en Colombia " in Corporacion Araracuara, Colonizacion del bosque humedo tropical, Bogota, 1988.

14. Soulignons que les narcotrafiquants ont massivement investi dans la terre. On estime qu'ils ont acquis environ 4 millions d'hectares, souvent parmi les plus fertiles, ce qui représente une véritable « contre-révolution agraire».

15. Cette commune située sur le rio Magdalena et proche de la région des émeraudes, la seule région économique d'importance nationale à avoir réussi à se soustraire grâce à ses puissants moyens « d'autodéfense » à l'influence des guérillas, a été le fief de Gonzalo Rodriguez Gacha à la fin des années 1980. Une école de formation paramilitaire y avait été installée avec le concours de mercenaires étrangers.

16. Corregimiento de Mutata, commune de l'Uraba. Les procédures de justice expéditive utilisées par les FARC y faisaient régner dès 1990 un climat de peur.

17. Cf. Adolfo Atehortua, El poder y la sangre. Las historias de Trujillo, Valle, Cali, Gobernacion del Valle, 1996.

18. Cf. la thèse d'Andres Penate, Oil and politics, Oxford, 1992.

19. Terme local pour désigner les interférences entre bandes de quartier et les principales organisations armées.

20. Comme les exemples de La India, San Jose de Apartado, de Riosucio ou d'Aguachica, frappées par les paramilitaires mais aussi par les guérillas, le démontrent.

21. Non-Lieux, Introduction à une Anthropologie de la Surmodernité, Paris, Seuil, 1992.

22. Bien des exemples m'ont été personnellement racontés de personnes exécutées à Barrancabermeja pour s'être rendues dans un quartier tenu par un autre groupe. Dans l'Uraba, des cas m'ont été également cités de personnes tuées par les FARC pour s'être rendues de Riosucio, à l'époque encore sous contrôle de la guérilla, à Turbo, déjà conquise par les militaires. Enfin un massacre commis l'an dernier dans la "communauté de paix» de Riosucio vient apparemment de ce que, par simple jalousie personnelle, des informateurs avaient donné de fausses indications aux paramilitaires sur la supposée sympathie des victimes envers les guérillas.

23. Au début de cette année, la presse faisait état d'un jeune paysan qui s'était engagé dans la guérilla à seule fin de pouvoir tuer un commandant responsable de l'assassinat de son père.

24. J'ai réalisé de nombreux entretiens avec des colons dans le Putumayo, il y a quelques années avec presque toujours des réponses en ce sens. 
25. CfAMolano, Los años del tropel, Bogota, CEREC-CINEP, 1985.

26. No nacimos pa' semilla, Bogota, CINEP, 1993.

27. La Virgen de los sicarios, Bogota, Alfaguara, 1994.

28. Les deux premiers étaient présidents de l'Union Patriotique, ce parti formé sous l'égide du Parti communiste et des FARC pendant les négociations avec le gouvernement de B. Betancur Luis Carlos Galan, leader du parti libéral, était le favori de l'élection présidentielle de 1990Carlos Pizarro, l'ancien chef du M19, s'était porté candidat à cette même élection. Alvaro Gomez Hurtado, fils de l'ancien président Laureano Gomez, représentant d'un conservatisme extrémiste, avait été l'une des deux principales figures du parti conservateur pendant trois décennies et avait été trois fois candidat à une élection présidentielle. Jaime Garzon était le plus populaire des humoristes de la télévision.

29. Cf. Zaki Laïdi, «L'urgence et la représentation de l'histoire », Esprit, 1998.

30. L'aspect complémentaire est le pardon facile attribué à celui qui reconnaît ses crimes. Bien des témoignages établissent que beaucoup des jeunes criminels de Medellin sont aisément pardonnés par les prêtres qui reçoivent leurs confessions. La transaction ne vaut pas seulement entre les humains, elle gouverne aussi les rapports avec le divin.

31. Par exemple entre 1905 à 1932, 1934 et 1946, et même entre 1964 et 1975.

32. Cf. D. Pécaut, « Passé, Présent et Futur de la Violence ", in J-M. Blanquer et C. Gros (dir.), La Colombie à l'aube du Troisième Millénaire, Paris, IHEAL, 1996.

33. Cf. G. Sanchez et alii, Colombia, Violencia y Democracia, Bogota, IEPRI, 1987, livre dans lequel les auteurs analysent la violence de la rue comme indépendante de la violence des organisations armées.

34. Sur l'identité narrative, cf. P. Ricœur, Le Soi et l'Autre, Paris, Seuil-Points, 1990. Ricœur distingue à propos de l'identité personnelle la «mêmeté ", qui renvoie à la permanence dans le temps de l'individu comme substance et l'« ipséité », qui renvoie aux dispositions durables à quoi se reconnaît une personne.

35. Je fais naturellement référence au livre de Robert Antelme.

36. L'impérialisme, Paris, Fayard, 1982, p.276.

37. Dans les entretiens que j'ai menés avec de jeunes démobilisés de l'EPL, c'est tout juste s'ils pouvaient citer Marx et Gaitan était loin d'être connu de tous. Certains étaient bien incapables de me fournir une représentation approximative du territoire colombien.

38. Cf. les analyses comparables sur la violence irlandaise de A. Feldman, Formations of Violence, The Narrative of the Body and Political Terror in Northern Ireland, Londres, The University of Chicago Press, 1991.

39. La forte abstention traditionnelle a reculé depuis quelque temps. Le vote devient un moyen de dire non à la violence. Et cela plus encore depuis que les guérillas en ont imposé le boycott en 1997 dans les régions qu'elles contrôlent.

40. Francisco Gutierrez Sanin, La ciudad representada, Bogota, Tercer Mundo-IEPRI, 1998.

\section{INDEX}

Index géographique : Amérique du Sud, Colombie

Mots-clés : forces paramilitaires, terreur, Théorie politique

Thèmes : FARC-EP 